\title{
Towards a LED based on a photonic crystal nanocavity for single photon sources at telecom wavelength
}

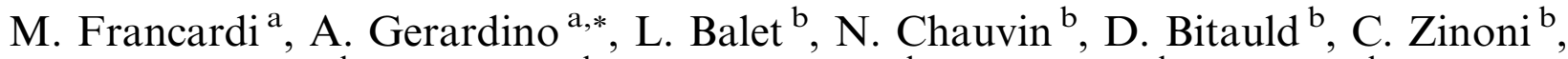 \\ L.H. Li ${ }^{\mathrm{b}}$, B. Alloing ${ }^{\mathrm{b}}$, N. Le Thomas ${ }^{\mathrm{b}}$, R. Houdré ${ }^{\mathrm{b}}$, A. Fiore ${ }^{\mathrm{b}}$ \\ a Institute for Photonics and Nanotechnologies-CNR, via Cineto Romano 42, 00156 Roma, Italy \\ ${ }^{\mathrm{b}}$ Ecole Polytechnique Fédérale de Lausanne (EPFL), Institute of Photonics and Quantum Electronics, CH-1015 Lausanne, Switzerland
}

Received 4 October 2007; received in revised form 16 December 2007; accepted 27 December 2007

Available online 12 January 2008

\begin{abstract}
A fundamental step towards achieving an "on demand" single photon source would be the possibility of electrical pumping for a single QD and thus the integration of such a device in an opto-electronic circuit. In this work we describe the fabrication process and preliminary results of a Light Emitting Diode (LED) to be integrated with a PhC nanocavity at telecom wavelength. We demonstrate the possibility of an effective electric pumping of the QDs embedded into the membrane by contacting the n-doped and p-doped layers of the thin membrane, which allows the fabrication of a $\mathrm{PhC}$ nanocavity on it.
\end{abstract}

(C) 2007 Elsevier B.V. All rights reserved.

Keywords: Photonic crystals; Optical nanocavities; LED; Single photon sources

\section{Introduction}

The technology of real single-photon devices is still in its infancy, while the physical basis for single-photon emission from single QDs seems well-established. The required telecom wavelength $(1300$ or $1550 \mathrm{~nm})$ poses significant challenges both in the epitaxial growth of the quantum dots (QDs) and in the measurement (InGaAs or Ge avalanche photodiodes must be used, with lower quantum efficiency and much higher noise). For these reasons, the few demonstrations of single-photon emission in the telecom bands [1-4] do not yet match the application requirements. An approach to the fabrication of efficient single-QD LEDs still has to be demonstrated [5], and a systematic investigation of the temperature limitations is missing. The enhancement of the spontaneous emission rate of an emitter on resonance with a mode of an optical

\footnotetext{
${ }^{*}$ Corresponding author. Tel.: +390641522242 ; fax: +390641522220.

E-mail addresses: marco.francardi@ifn.cnr.it (M. Francardi), gerardino@ifn.cnr.it (A. Gerardino).
}

cavity (Purcell effect [6]), can be used to increase the efficiency of the source. Recently, using a modified L3 defect nanocavity (3 in-line missing holes) in a photonic crystal $(\mathrm{PhC})$ on a GaAs membrane with a single layer of low density $\left(5-7 \mathrm{dot} / \mu^{2}\right)$ QDs in its center, we obtained quality factors $\mathrm{Q}$ as high as 16500 and measured, in resonance conditions, a Purcell factor of 8 at $1300 \mathrm{~nm}$ for the first time $[7,8]$. On the basis of these achievements, we have developed an original design to integrate an LED device at $1300 \mathrm{~nm}$ with a $\mathrm{PhC}$ nanocavity. The fabrication process is very challenging and we demonstrate an effective electrical pumping of the QDs into the membrane both in case of high and low areal density QDs.

\section{Fabrication process}

The light source at $\lambda=1300 \mathrm{~nm}$ consists of InAs QDs grown by molecular beam epitaxy (MBE) $[9,10]$. The LEDs have been fabricated on two heterostructures grown on a GaAs substrate: the first one has 3 layers of high QDs density $\left(\sim 300\right.$ dots $\left./ \mu \mathrm{m}^{2}\right)$ emitting at $1300 \mathrm{~nm}$ 
at room temperature $(R T)$; the second one has a single layer of low QDs density (5-7 dots $/ \mu \mathrm{m}^{2}$ ) emitting at $1300 \mathrm{~nm}$ at $5 \mathrm{~K}$. The QDs were grown at the center of a $320 \mathrm{~nm}$-thick GaAs membrane on top of a $1500 \mathrm{~nm}$-thick $\mathrm{Al}_{0.7} \mathrm{Ga}_{0.3} \mathrm{As}$ sacrificial layer and the doped layers are contained in the membrane. The main idea is to inject electrons from a top annular contact and holes from the sides of the mesa, using highly-doped GaAs contact layers to spread the current throughout the mesa to the center of the cavity. Recombination will occur on the entire surface of the mesa but the emission from the cavity center is enhanced and can be isolated with a combination of spatial and spectral filtering.
The fabrication process is based on e-beam lithography (EBL) (Vistec, EPBG 5HR working at $100 \mathrm{kV}$ ) and thinfilm techniques. The first step consists in patterning of the top, ring-shaped n-contact by lift-off of a multilayer of $\mathrm{Ni} / \mathrm{Ge} / \mathrm{Au} / \mathrm{Ni} / \mathrm{Au}$. The pattern is transferred by EBL on a $1 \mu \mathrm{m}$ thick UVIII resist. The large thickness of this resist layer allows us to lift-off the metal contact, deposited by gun-evaporation $(\mathrm{Ni} / \mathrm{Ge})$ and thermal evaporation $(\mathrm{Au})$ for a total thickness of $155 \mathrm{~nm}$. The contact is annealed at $400{ }^{\circ} \mathrm{C}$ for $30 \mathrm{~min}$ with a thermal ramp of $5{ }^{\circ} \mathrm{C} / \mathrm{min}$. Together with the contacts, markers to be used for the alignment of the successive exposures are also deposited. Then, we expose mesa patterns with different diameters
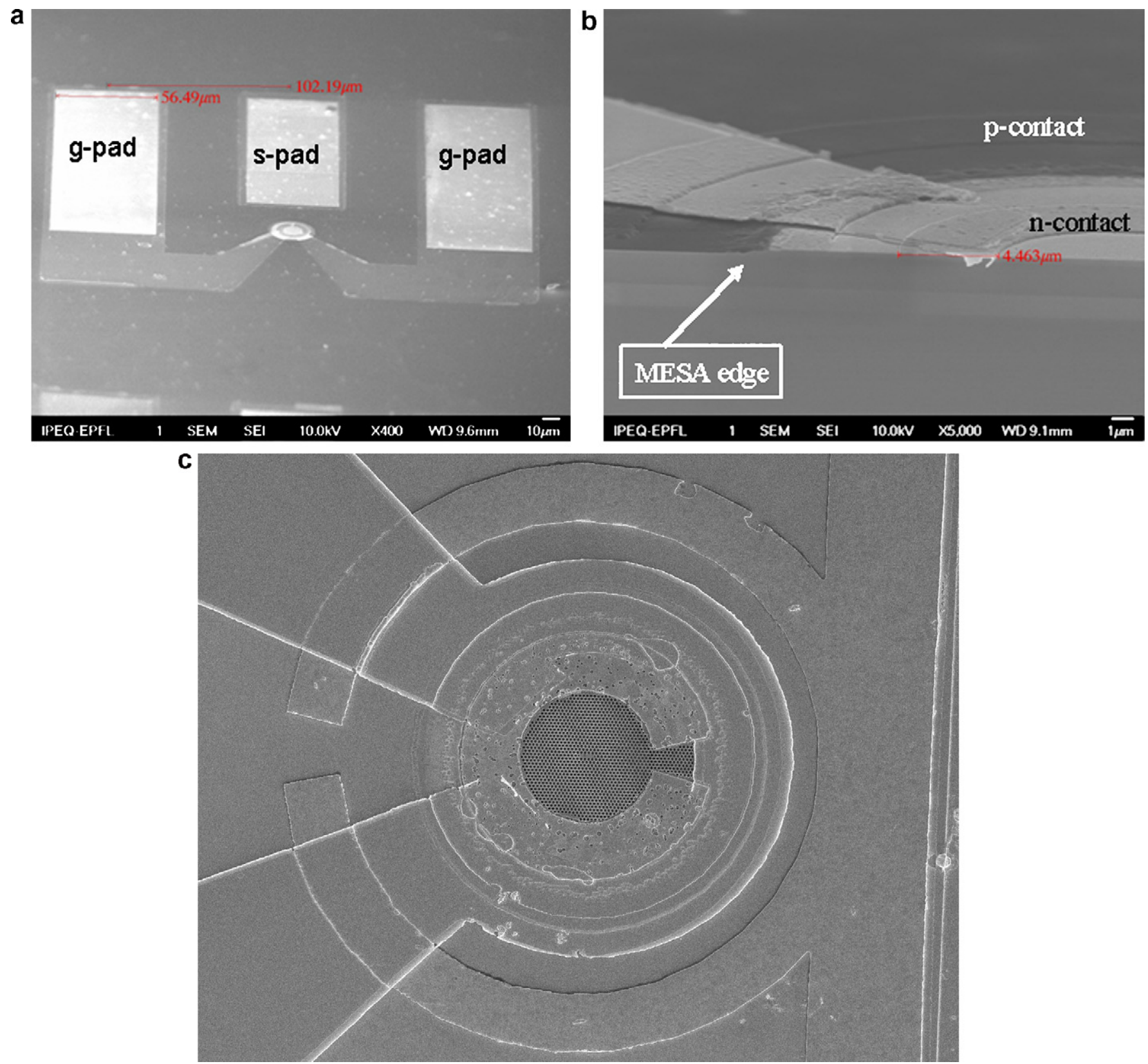

Fig. 1. (a) Top view (SEM image) of a LED at the end of the fabrication process, s-pad: signal pad, g-pad: ground pad; (b) same device type in section (after cleavage); (c) particular of the device with a $\mathrm{PhC}$ at the end of the process. 
(32-26-20-15-10 $\mu \mathrm{m}$ ) on a $300 \mathrm{~nm}$-thick HSQ (hydrogen silsesquioxane) resist and etch the mesa using a $\mathrm{H}_{3} \mathrm{PO}_{4}: \mathrm{H}_{2} \mathrm{O}_{2}: \mathrm{H}_{2} \mathrm{O}=3: 1: 30$ solution to obtain a height between $290 \mathrm{~nm}$ and $330 \mathrm{~nm}$. This wet etching process is the most crucial step of our fabrication process: we need to stop the wet etching exactly on the p-region of our membrane. The membrane is composed by layers of GaAs (n and $p$ doped) with AlGaAs layers in between, so the etching rate is not constant: to reach reproducible results we have to optimise this step accurately. The HSQ layer is then removed with a HF based solution. The p-contact is also shaped in a ring, in order to obtain the best hole injection around the mesa and it consists of $110 \mathrm{~nm} \mathrm{Ti} / \mathrm{Au}$ evaporated on a patterned UVIII layer and subsequent lift-off in acetone. A $200 \mathrm{~nm}$-thick $\mathrm{Si}_{3} \mathrm{~N}_{4}$ layer is deposited by PEC$\mathrm{VD}$ to create an insulating layer between the $\mathrm{n}$ and $\mathrm{p}$-contacts. The $\mathrm{Si}_{3} \mathrm{~N}_{4}$ is then removed by selective reactive ion etching (RIE) $\left(50 \mathrm{sccm} \mathrm{CHF}_{3}, 10 \mathrm{sccm} \mathrm{O}_{2}\right.$, pressure: 55 mTorr, power: $200 \mathrm{~W}$ ) from the n-contact on the top of the mesa and from part of the p-contact. By lift-off of a $\mathrm{Cr} / \mathrm{Au}$ (thickness: $110 \mathrm{~nm}$ ) layer we can connect the n-contact on the top of the mesa with the ground pads on the $\mathrm{Si}_{3} \mathrm{~N}_{4}$ surface by two bridge contacts. In the same process step we also connect the p-contact with the signal pad on the $\mathrm{Si}_{3} \mathrm{~N}_{4}$ layer. In order to carry out a continuous film over the mesa lateral edge we performed two tilted $\mathrm{Cr} / \mathrm{Au}$ evaporations. The lift-off step ends the fabrication of the LED. Fig. 1a shows a SEM image of a LED (mesa diameter: $32 \mu \mathrm{m})$ at this stage of the process. In Fig. $1 \mathrm{~b}$ a cleaved section of a device of the same type is shown. It is possible to see the $\mathrm{n}$-contact onto the mesa and the bridge that brings the $\mathrm{n}$-contact down on the sample. The anular p-contact under the $\mathrm{Si}_{3} \mathrm{~N}_{4}$ layer should also be noted. To integrate the $\mathrm{PhC}$ nanocavity on the LED a $150 \mathrm{~nm}$-thick $\mathrm{SiO}_{2}$ layer has been deposited by ECR-PECVD on the top of the substrate. The PhC pattern is transferred by EBL on a $200 \mathrm{~nm}$ thick PMMA resist and the usual process to fabricate $\mathrm{PhC}$ nanocavities has been performed [11]. In Fig. 1c is shown a particular of the mesa with a PhC cavity transferred on its surface at the end of the process.

\section{Results}

The electro-optical characterization of the LED has been performed in a cryogenic elettro-probe station coupled with a microelectroluminescence $(\mu \mathrm{EL})$ setup. The sample is mounted on a cold-finger cooled by a holder dipped in liquid $\mathrm{He}$ (about $3 \mathrm{~K}$ ). The optical emission from the top of devices can escape from the cryogenic set up through a window and is collected by a microscope objective (numerical aperture 0.3). A mirror reflects the InfraRed (IR) radiation (which is sent to an IR camera or focused into an optical-fiber and sent to the spectrometer) and transmits the visible radiation (that allows to observe the samples surface by a CCD camera). The electroluminescence (EL) is dispersed into a $1 \mathrm{~m}$ focal length monochromator equipped with a cooled InGaAs photodiode array detector; the spectral resolution of the setup is better than $30 \mu \mathrm{eV}(\approx 0.04 \mathrm{~nm})$.

The first tests have been carried out on high density InAs QDs samples emitting at $1300 \mathrm{~nm}$ at RT. The I-V curves measured on these devices (mesa area: $\sim 1400 \mu \mathrm{m}^{2}$ ) show typical rectifying $\mathrm{p}-\mathrm{n}$ junction characteristics with a voltage threshold in direct polarization of $\approx 1.5 \mathrm{~V}$. At low temperatures, a lower forward-bias current is measured, presumably due to reduced thermal activation through contact and heterostructure barriers. Nevertheless, the current injected through the junction is in the range of $\mathrm{mA}$, for an applied voltage $\geqslant 4 \mathrm{~V}$, sufficient to excite the InAs QDs in the active region. In fact EPL signal has been measured (Fig. 2a). From the IR camera image of a device under electrical injection we observe (Fig. 2b) a large emission from the edges of the mesa as well as from the center, attributed to scattering of light propagating in a waveguide mode within the mesa. So, we have processed the sample with low density InAs QDs, implementing a gold cover around the mesa border to reflect the scattered light to the sub-

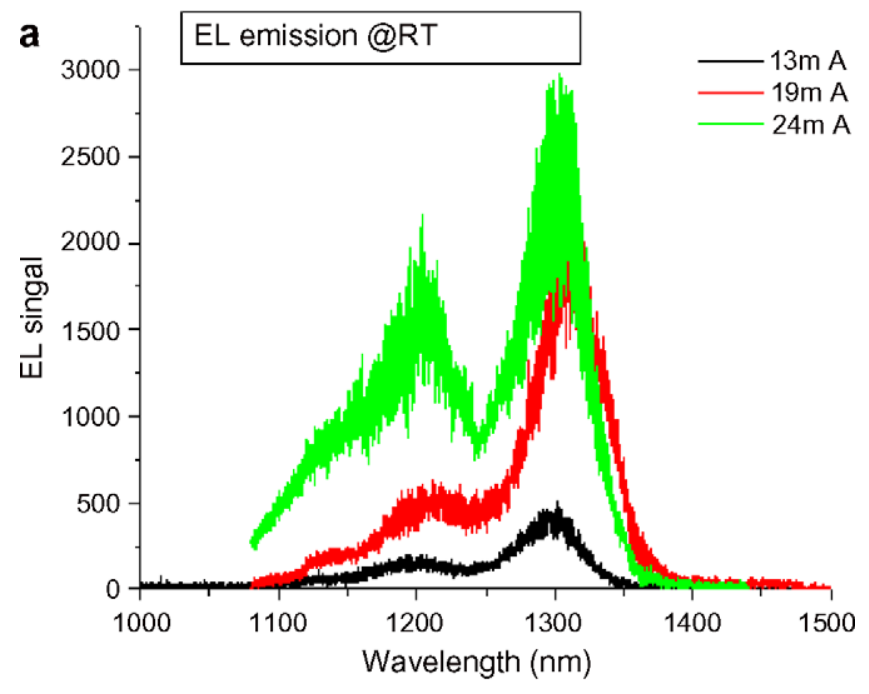

b

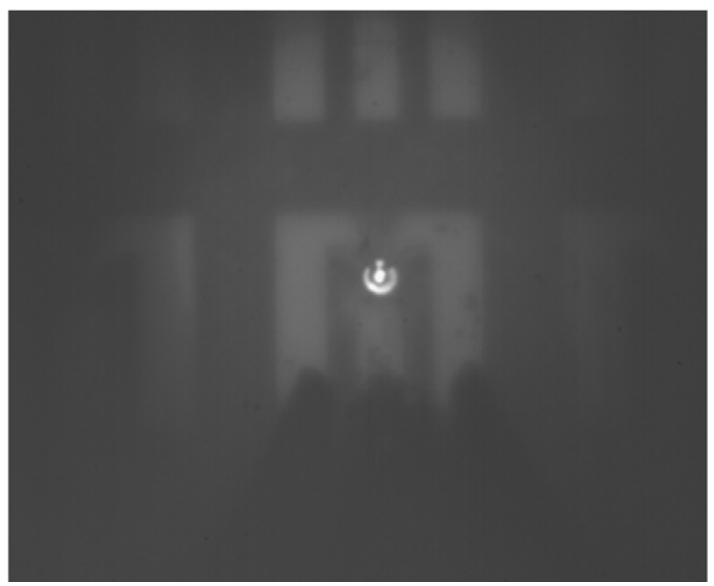

Fig. 2. (a) EPL emission ( $R T$ ) from a high density QD based LED for different pumping currents. (b) Picture from an IR camera of a high density QD LED under electrical injection. Note the light emitted from the mesa edges. 
a
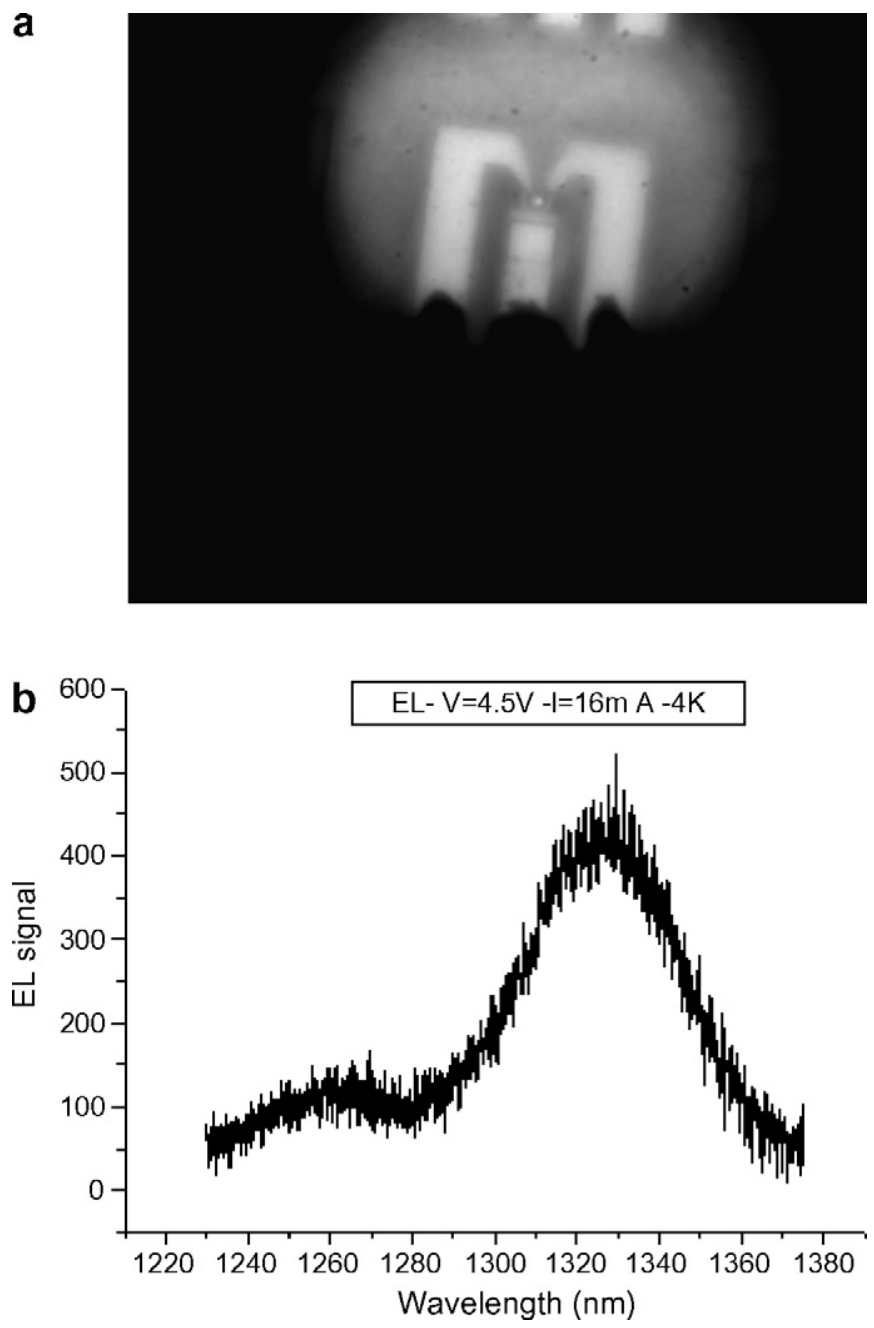

Fig. 3. (a) EL emission from the same LED at 4 K. (b) Picture from an IR camera of a low density QD LED under electrical injection. strate side. Fig. 3a shows the IR camera picture of a working device: the emission comes from the proper region and also the measured EPL spectum (Fig. $3 b$ ) is in agreement with the PL signal of the same sample. The measurements on the low density samples have been performed at $4 \mathrm{~K}$.

\section{Conclusions}

We have presented a LED structure working at $1300 \mathrm{~nm}$, designed to be integrated with a $\mathrm{PhC}$ nanocavity. First steps in this last direction have been already performed, optimizing the exposure process of the $\mathrm{PhC}$ nanocavities on the LED top and their transfer into the membrane. Our next goals will be to demonstrate the coupling of InAs QDs at $1300 \mathrm{~nm}$ to a PhC cavity mode, also in electrical pumping conditions.

\section{Acknowledgements}

Swiss National Science Foundation, Italian MIURFIRB program, the FP6 NoE "ePIXnet", EU project "QAP", SER-COST program.

\section{References}

[1] M. Takemoto et al., Jpn. J. Appl. Phys. 43 (2004) L349-L351.

[2] M.B. Ward et al., Appl. Phys. Lett. 86 (2005) 201111.

[3] C. Zinoni et al., Appl. Phys. Lett. 88 (2006) 131102.

[4] C. Zinoni et al., Appl. Phys. Lett. 91 (2007) 031106.

[5] H.G. Park et al., Science 305 (2004) 1444-1447.

[6] E.M. Purcell, Phys. Rev. 69 (1946) 681.

[7] M. Francardi et al., Phys. Status Solidi (c) 3 (11) (2006) 3693-3696.

[8] L. Balet et al., Appl. Phys. Lett. 91 (2007) 123115.

[9] B. Alloing et al., Appl. Phys. Lett. 86 (2005) 101908.

[10] W.H. Chang et al., Phys. Rev. Lett. 96 (2006) 117401.

[11] A. Gerardino et al., Microeletron. Eng. 84 (2007) 5-8. 